\title{
Rasgos de personalidad y consumo de alcohol en estudiantes universitarios
}

\section{Personality traits and alcohol consumption in university students}

\section{Características da personalidade e consumo de álcool em estudantes universitários}

\author{
Julia Alejandra Sifuentes-Castro ${ }^{1}$ \\ (D) https://orcid.org/0000-0002-2834-1785 \\ Manuel Antonio Lopez-Cisneros ${ }^{2 *}$ \\ (iD https://orcid.org/0000-0002-9384-5752 \\ Francisco Rafael Guzmán-Facundo 3 \\ (iD) https://orcid.org/0000-0002-6951-8989 \\ Juan Yovani Telumbre-Terrero ${ }^{4}$ \\ (iD) https://orcid.org/0000-0002-8695-7924 \\ Pedro Moisés Noh-Moo 5 \\ https://orcid.org/0000-0003-1580-5533
}

1. Estudiante del Programa de Maestría en Ciencias de Enfermería. Universidad Autónoma de Nuevo León. Facultad de Enfermería.

2. Doctor en Ciencias de Enfermería. Universidad Autónoma del Carmen. Facultad de Ciencias de la Salud. Hospital General de Zona No. 4 del Instituto Mexicano del Seguro Social.

3. Doctor en Ciencias de Enfermería. Universidad Autónoma de Nuevo León. Facultad de Enfermería.

4. Maestro en Ciencias de Enfermería. Universidad Autónoma del Carmen. Facultad de Ciencias de la Salud.

5. Estudiante del Programa de Maestría en Ciencias de Enfermería. Universidad Autónoma de Sinaloa. Facultad de Enfermería Mochis.

*Autor para correspondencia: mlcisneros@hotmail.com

\section{Resumen}

Introducción: Los rasgos de personalidad son patrones de conducta persistentes en la forma de pensar, percibir y relacionarse, por lo que fungen como condicionantes que pueden hacer vulnerable al individuo para adquirir conductas saludables o nocivas para la salud, como el consumo de drogas lícitas e ilícitas. Objetivo: Determinar la relación entre los rasgos de personalidad y el consumo de alcohol en estudiantes universitarios de Ciudad del Carmen Campeche, México. Metodología: Estudio cuantitativo, no experimental, descriptivo correlacional, transversal, efectuado en una muestra de 238 estudiantes universitarios del área de la salud. Resultados: La prevalencia lápsica del consumo de alcohol, se presentó en mayor proporción (85.3\%), en comparación a la prevalencia actual (39.9\%) e instantánea (20.6\%). En cuanto al tipo de consumo de alcohol, existe un consumo dependiente (19.9\%) en estudiantes universitarios, a pesar de predominar un consumo de riesgo (60.2\%). De acuerdo con el AUDIT, se encontró una relación positiva con el rasgo de personalidad de 
extraversión ( $r s=.165, p=.02$ ). Conclusiones: Se identificó el perfil del consumo de alcohol de los jóvenes, así como una relación del rasgo de personalidad extraversión con el consumo de alcohol, lo cual pone en evidencia la necesidad del diseño e implementación de intervenciones de Enfermería eficientes y oportunas que contribuyan de manera significativa a la disminución y erradicación de hábitos nocivos para la salud y el fomento de estilos de vida saludables.

Palabras clave: Consumo de Bebidas Alcohólicas; Adulto Joven; Personalidad (DeCS).

\begin{abstract}
Introduction: Personality traits are persistent patterns of behavior in the way of thinking, perceiving and relating, so they serve as determining factors that can make the individual vulnerable to acquire behaviors that are healthy or harmful to health, such as the use of licit drugs and illicit. Objective: To determine the relationship between personality traits and alcohol consumption in university students from Ciudad del Carmen Campeche, Mexico. Methodology: Quantitative, nonexperimental, descriptive correlational, cross sectional study, carried out on a sample of 238 university students in the health area. Results: The lapsed prevalence of alcohol consumption was presented in a higher proportion (85.3\%), compared to the current prevalence (39.9\%) and instantaneous (20.6\%). Regarding the type of alcohol consumption, there is a dependent consumption (19.9\%) in university students, despite the prevalence of risky consumption (60.2\%). According to AUDIT, a positive relationship was found with the extraversion personality trait ( $r s=.165, p=.02$ ). Conclusions: The profile of alcohol consumption in young people was identified, as well as a relationship of the extraversion personality trait with alcohol consumption, which highlights the need for the design and implementation of efficient and timely nursing interventions that contribute significantly to the reduction and eradication of harmful habits for health and the promotion of healthy lifestyles.
\end{abstract}

Key words: Alcohol Drinking; Young Adult; Personality.

\begin{abstract}
Abstrato
Introdução: Os traços de personalidade são padrões persistentes de comportamento na maneira de pensar, perceber e se relacionar; portanto, servem como fatores condicionantes que podem tornar o indivíduo vulnerável a adquirir comportamentos saudáveis ou prejudiciais à saúde, como o uso de drogas lícitas e ilícito. Objetivo: Determinar a relação entre traços de personalidade e consumo de álcool em estudantes universitários de Ciudad del Carmen Campeche, México. Metodologia: Estudo quantitativo, não experimental, correlacional, descritivo, transversal, realizado em uma amostra de 238 universitários da área da saúde. Resultados: A prevalência decorrida de consumo de álcool foi apresentada em uma proporção maior (85,3\%), em comparação à prevalência atual $(39,9 \%)$ e instantânea $(20,6 \%)$. Quanto ao tipo de consumo de álcool, existe um consumo dependente (19,9\%) em estudantes universitários, apesar da prevalência de consumo arriscado $(60,2 \%)$. Segundo o AUDIT, foi encontrada uma relação positiva com o traço de personalidade extroversiva ( $r s=0,165, p=$ 0,02). Conclusões: Identificou-se o perfil do consumo de álcool em jovens, bem como uma relação do traço de personalidade extroversão com o consumo de álcool, o que evidencia a necessidade de desenho e implementação de intervenções de enfermagem eficazes e oportunas que contribuam significativamente à redução e erradicação de hábitos nocivos à saúde e à promoção de estilos de vida saudáveis.
\end{abstract}

Palavras-chave: Consumo de Bebidas Alcoólicas; Adulto Jovem; Personalidade (DeCS).

Recibido: 03/04/2020

Aceptado: $25 / 06 / 21$ 


\section{Introducción}

La conducta de consumo de alcohol se considera uno de los principales retos que enfrenta actualmente la salud pública, debido a que es un problema de salud de origen multifactorial, que condiciona una gama de trastornos biológicos, psicológicos y sociales, entre los que se encuentran: diversos tipos de cáncer, cirrosis hepática, problemas cardiovasculares, trastornos mentales, alteración de las relaciones interpersonales y accidentes de tránsito, entre otros, siendo responsable de alrededor de 3.3 millones de muertes al año en el mundo, impactando significativamente en el crecimiento, desarrollo y fortalecimiento del núcleo familiar y social, limitando no solo al consumidor sino a todo el contexto en el que se desenvuelve, afectando la calidad de vida del individuo, familia grupo y/o comunidad ${ }^{(1,2)}$.

Uno de los grupos etarios con mayor vulnerabilidad para adquirir conductas de riesgo, es el conformado por adolescentes y jóvenes, debido a que, durante esta etapa de crecimiento y desarrollo, el individuo se enfrenta a cambios biopsicosociales en el contexto familiar, escolar y laboral, (3-6). En México existen hallazgos estadísticos que señalan que los adolescentes y jóvenes con edades comprendidas entre los 18 a 25 años ingieren grandes cantidades de alcohol por ocasión de consumo. Se ha estimado una prevalencia de consumo de alcohol alguna vez en la vida entre $77.1 \%$ a $77.3 \%$, el último año entre $55.7 \%$ a $53.3 \%$, el consumo en el último mes entre $35.0 \%$ a $39.9 \%$ y en la última semana entre $13.9 \%$ a $22.1 \%$. Estos datos ponen en evidencia el aumento considerable en el consumo excesivo de bebidas embriagantes, ya que en el último mes y última semana está por encima de la media nacional (19.8\%), predominantemente en la región sureste del país ${ }^{(7,8)}$.

Uno de los condicionantes más relacionados con el consumo de alcohol durante la juventud son los rasgos de la personalidad, definidos como patrones de conductas persistentes, en la forma de pensar, percibir y relacionarse de una persona ${ }^{(9,10)}$. Por lo que McCrae y Costa ${ }^{(11)}$, propusieron un Modelo de cinco grandes dimensiones de la personalidad (neurotismo, extraversión, apertura, amabilidad y conciencia), los cuales permiten comprender la personalidad de los individuos para la adquisición de hábitos que influyen para desarrollar, mantener o eliminar comportamientos y estilos de vida saludables y no saludables. 
En el fenómeno de la drogodependencia, el rasgo de personalidad neurotismo involucra a individuos con baja estabilidad emocional y ha sido asociado fuertemente con una mayor conducta de consumo de drogas lícitas e ilícitas en jóvenes ${ }^{(12,13)}$, mientras que la extraversión hace referencia a individuos sociables, con afectividad positiva, en búsqueda de aventuras e impulsividad, y estudios realizados en Estados Unidos de América y Serbia han relacionado este rasgo con un mayor consumo de drogas $(5,14)$.

Respecto al rasgo de personalidad apertura y amabilidad, éste se caracteriza por individuos con ideas asertivas y libertad de pensamiento, con capacidad de altruismo, identificados por su compasión, confianza y generosidad, sin embargo, investigaciones efectuadas en España han evidenciado una asociación de dicho rasgo de personalidad con la ingesta de bebidas embriagantes en jóvenes ${ }^{(7,15)}$. Así mismo el rasgo de personalidad conciencia muestra la cualidad de un elevado sentido del deber, son individuos diligentes, responsables y disciplinados, siendo un factor protector para un menor consumo de alcohol ${ }^{(12,16)}$.

A pesar de las diversas características de pensamientos, sentimientos y comportamientos que caracteriza a los jóvenes, los rasgos de personalidad en relación con las adicciones se direccionan a desarrollar diversos problemas universales, principalmente en los estudiantes universitarios, quienes además de estar transitando por los cambios propios de la juventud, también como futuros profesionales se encuentran inmersos en un proceso de formación académica que se origina en un contexto sociocultural que puede servir como factor protector o en ocasiones como un factor de riesgo hacia la conducta de consumo de alcohol $(8,16,17,18)$. Aunado a que dentro del proceso de formación, el estudiante universitario se enfrenta a múltiples desafíos escolares, que ocasionan alteraciones vinculadas con el cansancio emocional, el agotamiento físico, la abrumadora carga horaria, el aumento de la responsabilidad, la limitación de la convivencia familiar, la carencia de actividades recreativas y de diversión, que se convierten en factores que condicionan la probabilidad de adquirir conductas nocivas para la salud, relacionadas con el consumo de alcohol como remedio para mitigar el estrés académico y las situaciones antes mencionadas ${ }^{(18,19)}$.

Por tal motivo es importante tener un acercamiento a este fenómeno de estudio, vinculado con los rasgos de personalidad, que permita obtener mayores conocimientos sobre patrones de conductas y su relación con posibles 
situaciones de riesgo ${ }^{(20)}$. En este sentido la presente investigación tuvo como objetivo determinar la relación entre los rasgos de personalidad y el consumo de alcohol en estudiantes universitarios.

\section{Metodología}

Estudio con enfoque cuantitativo, no experimental, correlacional y transversal, dado que se estableció la relación existente entre los rasgos de personalidad y el consumo de alcohol (21). La población de interés estuvo conformada por jóvenes estudiantes universitarios del área de la salud pertenecientes a una institución pública de educación superior ubicada en Ciudad del Carmen, Campeche, México. El muestreo fue aleatorio estratificado por semestre, estimándose el tamaño de muestra mediante el paquete estadístico n'Query Advisor versión $7.0^{(22)}$, con una potencia del $90 \%$, significancia de .05 , correlación de .30 , tasa de no respuesta del $10 \%$; quedando una muestra de 238 participantes.

Para la recolección de la información, se obtuvo el registro FCS/032018 ante el Comité de Investigación de la Facultad de Ciencias de la Salud de la Universidad Autónoma del Carmen, así como, la autorización de la institución educativa donde se realizó el proyecto, se explicó a los directivos el objetivo del estudio, solicitando la lista actual de alumnos inscritos en cada semestre de acuerdo al ciclo escolar vigente, para seleccionar a los participantes de manera aleatoria, se invitó a los estudiantes elegidos, para participar de manera voluntaria y anónima, a quienes aceptaron se les entrego el consentimiento informado para obtener su autorización.

La aplicación de los instrumentos se realizó el día y horario acordado con los estudiantes, entregándose estos en un sobre cerrado, el cual abrieron de forma individual, para dar lectura únicamente al objetivo del estudio y las instrucciones de llenado, en todo momento se respetó la integridad de los participantes, apegado a lo establecido en la Ley General de Salud en Investigación con seres humanos ${ }^{(23)}$, al finalizar cada alumno colocaba el sobre en una urna, ubicada a la salida del aula.

Se utilizaron tres instrumentos de lápiz y papel que fueron: a) Cédula de Datos Personales y Prevalencias del Consumo de Alcohol (CDPPCA), b) Cuestionario de Identificación de Trastornos por Consumo de Alcohol (AUDIT), desarrollado por la Organización Mundial de la Salud ${ }^{(24)}$, validado en población mexicana por De la Fuente \& 
Kershenobich ${ }^{(25)}$ y c) Inventario de Personalidad Reducido de Cinco Factores (NEO FFI) ${ }^{(26)}$, que se deriva del Revised NEO Personality Inventory (NEO-PI-R)(27) y validado en población mexicana por Reyes, Álvarez, Peredo, Miranda y Rebolledo (28).

La Cédula de Datos Personales y Prevalencias de Consumo de Alcohol (CDPPCA), recaba información general vinculada con datos personales del estudiante universitario como: edad, género, semestre, programa educativo que cursa, además de prevalencias de consumo de alcohol, clasificadas en el consumo de alguna vez en la vida, en último año, último mes, últimos siete días, así como la edad de inicio de consumo de alcohol y el número de copas que ingiere el joven en un día típico.

La Prueba de Identificación de Trastornos derivados del Consumo de Alcohol (AUDIT), consta de 10 preguntas de opción múltiple con un rango de puntuación de 0 a 4, permite identificar a los individuos con problemas del uso y abuso del alcohol durante el último año. Las puntuaciones de la escala fluctúan entre 0 a 40, donde a mayor puntuación existe mayor consumo ${ }^{(25)}$. El AUDIT consta de tres dominios, los reactivos 1, 2 y 3 indican la cantidad y frecuencia del consumo de alcohol y determinan el consumo sensato (sin riesgo), los reactivos 4 al 6 evalúan la posibilidad de que exista dependencia al consumo de alcohol (de riesgo) y los reactivos 7, 8, 9 y 10 exploran el consumo dañino de alcohol (perjudicial). El AUDIT en el presente estudio obtuvo un Alpha de Cronbach de .79.

El Inventario de Personalidad Reducido de Cinco Factores (NEO FFI), se constituye de 60 reactivos clasificados en cinco dimensiones (neurotismo, extraversión, apertura, amabilidad, conciencia), cada dimensión se conforma de 12 ítems, con puntuaciones que van desde 0 hasta 4, en una escala de puntuación donde $0=$ es totalmente desacuerdo, 1 = es en desacuerdo, 2 = es neutral, 3 = es de acuerdo y 4 = es totalmente de acuerdo. Las puntuaciones se obtienen sumando las respuestas de los 12 reactivos de cada dimensión, haciendo un valor total que va de 0 a 48 puntos, lo que significa que a mayor puntaje mayor es la característica de personalidad definida por la dimensión de tendencia. EI NEO FFI en esta investigación reportó un Alpha de Cronbach de .72.

El proceso estadístico de la información obtenida (captura, análisis e interpretación de los resultados) se efectúo a través del programa estadístico Statistical Package for the Social Sciences (SPSS) versión 23.0 para Windows, 
haciendo uso de estadística descriptiva (frecuencias, proporciones, medidas de tendencia central y dispersión) e inferencial. Se determinó la confiabilidad de los instrumentos a través del Alpha de Cronbach, así como la prueba de normalidad Kolmogorov - Smirnov y dado los resultados de estudio se decidió utilizar pruebas no paramétricas (Prueba U de Mann Whitney y Correlación Spearman).

\section{Resultados}

El $66.4 \%$ de los estudiantes universitarios pertenecen al género femenino, con una media de edad de 21.24 años ( $D E$ = 2.9), referente al grado escolar predominó que el $37.0 \%$ está cursando el segundo semestre y $31.9 \%$ el octavo semestre, en relación con la pertenencia a los programas educativos de la Facultad de Ciencias de la Salud, 26.9\% de los participantes están inscritos en la Licenciatura en Enfermería, seguido de la Licenciatura en Medicina con 25.6\%, posteriormente la Licenciatura en Nutrición con 14.7\%, Licenciatura en Psicología Clínica con 13.4\%, Licenciatura en Fisioterapia con 11.8\% y Licenciatura en Educación Física y Deporte con 7.6\%.

Respecto al consumo de alcohol, se comprobó que la media de edad de inicio de consumo de alcohol fue a los 17.5 años $(D E=2.3)$, con un promedio de consumo de bebidas embriagantes en un día típico de 4.1 copas $(D E=$ 3.5), siendo la cerveza la bebida de mayor preferencia en los estudiantes universitarios (49.2\%), seguido de bebidas alcohólicas preparadas (15.2\%).

En relación con las prevalencias del consumo de alcohol en los estudiantes universitarios, la Tabla 1, muesta que la prevalencia lápsica se presentó en mayor proporción (85.3\%), en comparación a la prevalencia actual (39.9\%) e instantánea (20.6\%).

Tabla 1. Prevalencias del consumo de alcohol en estudiantes universitarios

\begin{tabular}{lllll}
\hline & \multicolumn{3}{l}{ Si } & \multicolumn{3}{l}{ No } \\
\cline { 2 - 5 } Prevalencia & $f$ & 85.3 & 35 & 14.7 \\
\hline Alguna vez en la vida & 203 & 80.3 & 47 & 19.7 \\
En el último año & 191 & 39.9 & 143 & 60.1 \\
En el último mes & 95 & 20.6 & 189 & 79.4 \\
En la última semana & 49 & & \\
\hline
\end{tabular}

Fuente: $n=238, f=$ frecuencia, $\%=$ porcentaje. 
En la Tabla 2 se evidencia que existe un consumo dependiente (19.9\%) en los estudiantes, a pesar de predominar el consumo de riesgo o sensato (60.2\%).

Tabla 2. Tipos de consumo de alcohol de acuerdo con el AUDIT en estudiantes universitarios

\begin{tabular}{lllll}
\hline & \multicolumn{3}{c}{$\mathrm{Si}$} & No \\
\cline { 2 - 5 } Tipo de consumo & $f$ & $\%$ & $f$ & $\%$ \\
\hline Consumo sensato & 115 & 60.2 & 76 & 39.8 \\
Consumo dependiente & 38 & 19.9 & 153 & 80.1 \\
Consumo dañino & 62 & 26.1 & 129 & 67.5 \\
\hline
\end{tabular}

Fuente: $n=238, f=$ frecuencia, $\%=$ porcentaje.

En cuanto a los rasgos de personalidad se destaca que el rasgo de personalidad conciencia predomina con una media de $28.7(D E=7.4)$, donde el 34.9\% de los estudiantes afirmo tener objetivos claros y esforzarse por alcanzarlos de forma ordenada. Mientras que el rasgo de personalidad neurotismo obtuvo la media de $18.0(D E=8.4)$, se destaca que el $31.8 \%$ de los universitarios refirió poseer bastante estabilidad emocional, seguido del $26.2 \%$ que manifiesta estar en desacuerdo de realizar las cosas de manera impulsiva y luego se arrepienten (Tabla 3).

Tabla 3. Medidas de tendencia central y variabilidad del inventario NEO FF-I en estudiantes universitarios

\begin{tabular}{clllllll}
\hline $\begin{array}{l}\text { Escala total } \\
\text { dimensiones }\end{array}$ & $\mathbf{y}$ & $\tilde{X}$ & Mdn & $D E$ & $S^{2}$ & Valor Mín. & Valor Máx. \\
\hline NEO FF-I & 125.57 & 125.00 & 19.73 & 389.40 & 77.00 & 172.00 \\
Neurotismo & 18.07 & 17.00 & 8.42 & 71.03 & 2.00 & 46.00 \\
Extraversión & 26.37 & 26.00 & 9.08 & 82.59 & 7.00 & 45.00 \\
Apertura & 25.26 & 25.00 & 7.02 & 49.38 & 8.00 & 46.00 \\
Amabilidad & 27.10 & 27.00 & 7.31 & 53.46 & 7.00 & 45.00 \\
Conciencia & 28.76 & 28.00 & 7.48 & 55.99 & 13.00 & 47.00 \\
\hline
\end{tabular}

Fuente: $n=238, \tilde{X}=$ media, Mdn = mediana, $D E=$ desviación estándar, $S^{2}=$ Varianza, Valor Min = valor mínimo, Valor Máx $=$ valor máximo

Se identificó que existen diferencias significativas entre el consumo de alcohol alguna vez en la vida $(U=$ 2564.0, $p=.009)$ y en el último año $(U=3460.0, p=.015)$ con el rasgo de personalidad conciencia. Así mismo se encontraron también diferencias significativas entre consumo de alcohol en el último año $(U=3458.5, p=.015)$ y en el último mes $(U=5706.5, p=.037)$ con el rasgo de personalidad neurotismo (Tabla 4). 
Tabla 4. Prueba U de Mann - Whitney para las prevalencias de consumo de alcohol por rasgos de personalidad en estudiantes universitarios

\begin{tabular}{|c|c|c|c|c|c|c|}
\hline \multicolumn{2}{|c|}{ Consumo de alcohol alguna vez en la vida } & \multirow{2}{*}{$\begin{array}{l}n \\
203\end{array}$} & \multirow{2}{*}{$\begin{array}{r}\tilde{X} \\
28.25\end{array}$} & \multirow{2}{*}{$\begin{array}{l}\text { Mdn } \\
28.00\end{array}$} & \multirow{3}{*}{$\begin{array}{l}U \\
2564.00\end{array}$} & \multirow{3}{*}{$\begin{array}{l}p \\
.009\end{array}$} \\
\hline \multirow{2}{*}{ Conciencia } & $\mathrm{Si}$ & & & & & \\
\hline & No & 35 & 31.71 & 33.00 & & \\
\hline \multicolumn{7}{|c|}{ Consumo de alcohol en el último año } \\
\hline \multirow{2}{*}{ Neurotismo } & $\mathrm{Si}$ & 191 & 18.71 & 18.00 & \multirow{2}{*}{3458.50} & \multirow{2}{*}{.015} \\
\hline & No & 47 & 15.44 & 15.00 & & \\
\hline \multirow{2}{*}{ Conciencia } & $\mathrm{Si}$ & 191 & 28.17 & 28.00 & \multirow{2}{*}{3460.00} & \multirow{2}{*}{.015} \\
\hline & No & 47 & 31.12 & 31.00 & & \\
\hline \multicolumn{7}{|c|}{ Consumo de alcohol en el último mes } \\
\hline \multirow{2}{*}{ Neurotismo } & $\mathrm{Si}$ & 95 & 19.53 & 19.00 & \multirow{2}{*}{5706.50} & \multirow{2}{*}{.037} \\
\hline & No & 143 & 17.09 & 16.00 & & \\
\hline
\end{tabular}

Fuente: $n=238, \tilde{X}=$ media, $M d n=$ mediana, $U=$ Prueba $U$ de Mann Whitney, $p=$ significancia

Referente al objetivo de la investigación se determinó, una relación positiva y significativa entre el rasgo de personalidad de extraversión y el consumo de alcohol de acuerdo con al AUDIT ( $r s=.165, p=.023$ ).

Tabla 5. Correlación de Spearman para las variables cuantitativas del estudio

\begin{tabular}{cl}
\hline Variables & AUDIT \\
\hline \multirow{2}{*}{ Sumatoria NEO FF-I } & .023 \\
& .755 \\
\hline \multirow{2}{*}{ Neurotismo } & -.051 \\
Extraversión & .482 \\
\hline \multirow{2}{*}{ Apertura } & $.165^{\star}$ \\
Amabilidad & .023 \\
\hline \multirow{2}{*}{ Conciencia } & .006 \\
& .930 \\
\hline \multirow{2}{*}{ A } & -.045 \\
\hline
\end{tabular}

Nota: ${ }^{*} p=<.05,{ }^{* *} n=191,{ }^{* * *} n=238$. 


\section{Discusión}

El estudio permitió demostrar la relación existente entre los rasgos de personalidad y el consumo de alcohol en estudiantes universitarios de Ciudad del Carmen, Campeche, México, poniendo en evidencia que los universitarios, inician el consumo de alcohol en promedio a los 17 años de edad, datos semejantes a los reportados por la ENCODAT $(4,29)$, en un estudio realizado en estudiantes universitarios, que reportó el inicio de consumo de alcohol a los 17 años de edad o menos, lo cual podría vincularse principalmente a que los jóvenes se encuentran en un período de transición entre la adolescencia y la adultez, donde buscan la independencia, sintiéndose miembros y partícipes de sus propios comportamientos, valores, normas, principios, hábitos, costumbres y modas ${ }^{(8,17)}$.

Los hallazgos en esta investigación constataron que más de la cuarta parte de los universitarios, consumieron alcohol en el último mes, lo cual concuerda a lo reportado por investigaciones realizas en el Centro ${ }^{(17,30)}$ y Norte de México ${ }^{(8)}$, donde estipularon que el consumo de alcohol en los jóvenes universitarios, se debe a que son el sector de la población con más vulnerabilidad, debido a la gama de situaciones en las cuales los estudiantes universitarios se ven inmersos (ambiente interpersonal, entorno académico y contexto laboral), además que al llegar a la mayoría de edad, las posibilidades de accesibilidad a bebidas embriagantes aumentan exponencialmente, por lo que esta conducta de consumo se asocia también con la búsqueda de identidad, pertenencia a grupos y/o aceptación por pares, fenómenos sociales que incrementan el riesgo de consumo ${ }^{(31,32)}$.

La conciencia fue el rasgo de personalidad que destacó, debido a que los jóvenes están de acuerdo en trabajar mucho, para conseguir sus metas y tener claros sus objetivos, hallazgos que difieren de los resultados obtenidos por

investigaciones internacionales ${ }^{(7,15)}$, que señalan que los rasgos de personalidad que caracterizan a los jóvenes son neurotismo y extraversión, esto pudiera explicarse dado que los estudiantes universitarios que están enfocados al área de la salud, presentan un período de vida académico de mayor responsabilidad, ya que trabajan con seres humanos, por lo tanto su desempeño esta mediado por características de personalidad asociados a un elevado sentido del deber, responsabilidad y disciplina ${ }^{(5,19)}$. 
Además, la conciencia, fue el rasgo de personalidad que predomino en los estudiantes universitarios que no han consumido alcohol alguna vez en la vida y en el último año, hallazgos que se pueden atribuir a que es un rasgo de personalidad asociado a un alto sentido de responsabilidad, organización y control por sus actividades de la vida cotidiana (familiar, laboral, escolar), así como eficacia, eficiencia y autodisciplina, características determinantes para estilos de vida saludable ${ }^{(9,16)}$.

Por otro lado el rasgo personalidad neurotismo presentó mayor tendencia en estudiantes que refirieron haber consumido alcohol durante el último año y en el último mes, situación similar a lo reportado por un estudio realizado en estudiantes de posgrado de distintos países de Europa y América Latina ${ }^{(5)}$, lo cual se vincula con este rasgo de personalidad, caracterizado por personas con falta de ideas asertivas o positivas, afrontamiento ineficaz y pensamiento irracional, además que durante esta etapa de la vida el universitario se enfrenta a ambientes académicos y familiares que pueden provocar estrés, irritabilidad, ansiedad, cansancio y cambios en los patrones del sueño, potencializando el consumo de alcohol como una forma paliativa de disminuir estados emocionales negativos $(19,20)$.

En relación con el objetivo de esta investigación se comprobó una relación positiva y significativa del rasgo de personalidad extraversión con el consumo de alcohol, lo cual fue congruente con los hallazgos encontrados en investigaciones realizadas en España ${ }^{(12)}$ y Argentina ${ }^{(15)}$, quienes reportaron relación significativa entre el consumo de riesgo y perjudicial con el rasgo de personalidad extraversión, esto pudiera atribuirse a que los jóvenes universitarios con este rasgo de personalidad refirieron ser alegres, disfrutar las fiestas en las que están muchas personas, así como ser activos y estar donde hay acción, características condicionantes en su personalidad que aumentan la probabilidad de presentar un alto consumo de alcohol, lo cual puede afectar significativamente su calidad de vida.

\section{Conclusiones}

En conclusión podemos decir que los rasgos de personalidad contribuyen a obtener datos relevantes relacionados con el perfil del consumo de alcohol en los estudiantes universitarios del área de la salud, permitiendo conocer el impacto y trascendencia del fenómeno en este grupo etario, dando la oportunidad a que el profesional de Enfermería identifique de manera oportuna los factores de riesgo, permitiendo con esto el establecimiento a corto plazo de 
intervenciones específicas de la disciplina, direccionadas a la promoción y educación para la salud, que contribuyan a evitar, limitar o erradicar conductas vinculadas al consumo de sustancias lícitas e ilícitas, favoreciendo una mejor calidad de vida durante la edad adulta y fomentando estilos de vida saludables.

\section{Conflicto de intereses}

Todos los autores que participaron en el presente artículo manifiestan de común acuerdo, la inexistencia de algún conflicto de interés.

\section{Financiamiento}

Para el presente proyecto de investigación y publicación del artículo, se manifiesta que no hubo ningún tipo de financiamiento.

\section{Referencias bibliográficas}

1. Organización Mundial de la Salud. Alcohol [Internet]. OMS. 2018 [actualizado septiembre 2018; consultado Octubre 2019]. Disponible en: http://www.who.int/mediacentre/factsheets/fs349/es/

2. Organización Panamericana de la Salud. Regional Status Report on Alcohol and Health in the Americas [Internet]. OPS. 2015 [consultado Octubre 2019 ]. $\quad$ Disponible en http://www.paho.org/hq/index.php?option=com_content\&view=article\&id=11108\%3A2015-regional-reportalcohol-health\&catid $=1893 \% 3$ Anews\&ltemid $=41530$ \&lang $=$ es

3. Coleman J, Trunzo J. Personality, social stress, and drug use among college students. Psi Chi Journal of Psychological Research [Intenet]. 2015 [consultado Octubre 2019]; 20(1): 52-57. Disponible en: https://cdn.ymaws.com/www.psichi.org/resource/resmgr/journal_2015/Spring15JNColeman.pdf

4. Hernández-Cervantes Q, Alejandre-García J. Relación entre riesgo suicida y uso de tabaco o alcohol en una muestra de universitarios. Acta Universitaria [Internet]. 2015 [consultado Octubre 2019]; 25(2): 47-51. Disponible en: https://www.redalyc.org/pdf/416/41648310008.pdf

5. Motos P, Cortes MT, Giménez JA, Cadaveira F. Predictores del consumo semanal de alcohol y sus consecuencias asociadas en universitarios consumidores intensivos de alcohol. Adicciones [Internet]. 2015 [consultado Octubre 2019]; 27(2): 119-131. Disponible en: http://www.adicciones.es/index.php/adicciones/article/view/700/691

6. Telumbre-Terrero JY, Esparza-Almanza SE, Alonso-Castillo BA, Alonso-Castillo MTDJ. Actitudes hacia el consumo de alcohol y consumo de alcohol en estudiantes de enfermería. Investigación en Enfermería: Imagen y Desarrollo 
[Internet]. 2017 [consultado Octubre 2019]; 19(2): 69-81. Disponible en: https://www.redalyc.org/articulo.oa?id=145251406006

7. Instituto Nacional de Psiquiatría Ramón de la Fuente Muñiz, Instituto Nacional de Salud Pública, Comisión Nacional Contra las Adicciones, Secretaria de Salud. Encuesta Nacional de Adicciones 2011. Reporte de Alcohol [Internet]. 2012 [Consultado Octubre 2019]. Disponible en: https://encuestas.insp.mx/ena/ena2011/ENA2011_alcohol.pdf

8. Instituto Nacional de Psiquiatría Ramón de la Fuente Muñiz; Instituto Nacional de Salud Pública; Comisión Nacional Contra las Adicciones, Secretaria de Salud. Encuesta Nacional de Consumo de Drogas, Alcohol y Tabaco 2016- 2017. Reporte de Alcohol [Internet]. 2017 [Consultado Octubre 2019]. Disponible en: https://encuestas.insp.mx/ena/encodat2017/reporte_encodat_alcohol_2016_2017.pdf

9. Fehrman E, Muhammad AK, Mirkes EM, Egan V, Gorban AN. The Five Factor Model of personality and evaluation of drug consumption risk. Data Science [Internet]. 2017 [consultado Noviembre 2019]; 1(1): 231-242. Disponible en: https://link.springer.com/chapter/10.1007\%2F978-3-030-10442-9_1

10. American Psychiatric Association. Diagnostic and statistical manual of mental disorders [DSM-V]. 5ta ed. Estados Unidos. Editorial Médica Panamericana; 2013.

11. McCrae RR, Costa PT. The NEO Personality Inventory: Using the Five-Factor Model in Counseling. Journal of Counseling \& Development [Internet]. 1991 [consultado Noviembre 2019]; 69(4): 367-372. Disponible en: https://onlinelibrary.wiley.com/doi/abs/10.1002/j.1556-6676.1991.tb01524.x

12. Pilatti A, Urrizaga A, Chincolla A, Cupani M. Facetas de personalidades relacionada con el consumo elevado de alcohol en estudiantes universitarios de la provincia de Córdoba. Perspectivas en Psicología: Revista de Psicología y Ciencias Afines [Internet]. 2014 [consultado Noviembre 2019]; 11(1): 42-52. Disponible en: https://www.redalyc.org/pdf/4835/483547665006.pdf

13. Raketic D, Barisic JV, Svetozarevic SM, Gazibara T, Kisic D, Milovanovic SD. Five-Factor Model personality profiles: the differences between alcohol and opiae addiction among females. Psychiatria Danubina [Internet]. 2017 [consultado Noviembre 2019]; 29(1): 74-80. Disponible en: https://www.ncbi.nlm.nih.gov/pubmed/28291977

14. Hakulinen C, Elovainio M, Batty GD, Virtanen M, Kivimäki M, Jokela M. Personality and alcohol consumption: Pooled analysis of 72,949 adults from eight cohort studies. Drug \& Alcohol Dependence [Internet]. 2015 [consultado Diciembre 2019]; 151(1): 110-114. Disponible en: https://doi.org/10.1016/j.drugalcdep.2015.03.008

15. Gallego MS, Camacho L, Mezquita L, Viruela AM, Villa H, Ibañez MI, Ortet G. Relación de la personalidad y las conductas antinormativas de los amigos en el consumo de alcohol de los adolescentes. Àgora De Salut [Internet]. 2015 [consultado Diciembre 2019]; 1(1): 379-385. Disponible en: http://dx.doi.org/10.6035/AgoraSalut.2015.1.31 
16. McCrae RR, Costa PT. A contemplated revision of the NEO Five-Factor Inventory. Personality and individual differences [Internet]. 2004 [consultado Diciembre 2019]; 36(3): 587-596. Disponible en: https://doi.org/10.1016/S0191-8869(03)00118-1

17. Latorre-Román PÁ, Gallego-Rodríguez M, Mejía-Meza JA, García-Pinillos F. Consumo de tabaco y alcohol y práctica deportiva en universitarios mexicanos y españoles: asociación con la calidad de vida relacionada con la salud y la búsqueda de sensaciones. Gaceta Médica de México [Internet]. 2015 [consultado Diciembre 2019]; 151(1): 197-205. Disponible en: https://www.medigraphic.com/pdfs/gaceta/gm-2015/gm152i.pdf

18. Reguera-Torres ME, Sotelo-Cruz N, Barraza-Bustamante AG, Cortez-Laurean MT. Estilo de vida y factores de riesgo en estudiantes de la universidad de Sonora: Un estudio exploratorio. Boletín Clínico Del Hospital Infantil Del Estado de Sonora [Internet]. 2015 [consultado Enero 2020]; 32(2): 63-68. Disponible en: https://www.medigraphic.com/pdfs/bolclinhosinfson/bis-2015/bis152b.pdf

19. Oliveira EB, Furegato ARF. El trabajo del estudiante de enfermería como un factor de riesgo para el consumo de alcohol y otras drogas [Internet]. Revista Latino-am Enfermagem. 2008 [consultado Enero 2020]; 16(spe): 1-8. Disponible en: https://doi.org/10.1590/S0104-11692008000700010.

20. Gonzálvez MT, Espada JP, Guillon A, Secades R, Orgilés M. Asociación entre rasgos de personalidad y consumo de sustancias en adolescentes españoles. Adicciones [Internet]. 2016 [consultado Enero 2020]; 28(2): 108-115. Disponible en: http://www.adicciones.es/index.php/adicciones/article/view/777/733

21. Grove SK, Gray JF. Investigación en Enfermería: Práctica basada en la evidencia. 7a ed. Elsevier; 2019. 457 p.

22. Elashoff J, Dixon JW, Crede KM, Fotheringham N. nQuery Advisor (versión 7.0) Copyrigth [Software estadístico para PC]. Boston MA: Study Planning Software; 2007.

23. Secretaría de Salud. Ley General de Salud en Materia de Investigación para la Salud [Internet]. SS: 1987 [consultado Febrero 2020]. Disponible en: http://www.salud.gob.mx/unidades/cdi/nom/compi/rlgsmis.html

24. Babor TF, Higgins-Biddle JC, Saunders JB, Monteiro MG. AUDIT The Alcohol Use Disorders Identification Test: Guidelines for Use in Primary Care [Internet]. World Healt Organitation. 2a ed. Geneva; 2001 [consultado Febrero 2020]. Disponible en: https://apps.who.int/iris/handle/10665/67205

25. De la Fuente J R, Kershenobich D. El alcoholismo como problema médico. Revista de la Facultad de Medicina UNAM [Internet]. 1992 [consultado Febrero 2020]; 35(2): 47-51. Disponible en: http://revistas.unam.mx/index.php/rfm/article/view/74576/65953

26. Costa PT, McCrae RR. Manual Profesional de Inventario de Personalidad Neo Revisado [NEO PI-R]. Inventario Neo Reducido de Cinco Factores (NEO-FFI). 3a ed. Madrid: TEA Ediciones; 2008.

27. Costa PT, McCrae RR. Revised NEO personality inventory [PI-R] and NEO five-factor inventory [NEO-FFI]: Professional manual. Psychological Assessment Resources, Odesa, Florida; 1992. 
28. Reyes Zamorano, E., Álvarez Carrillo, C., Peredo Silva, A., Miranda Sandoval, A., \& Rebolledo Pastrana, I. M. (2014). Propiedades psicométricas del inventario de los Cinco Grandes en una muestra mexicana. Salud mental, 37(6), 491 497.

29. Barradas ME, Fernández N, Gutiérrez L. Prevalencia de consumo de alcohol en estudiantes. Revista Iberoamericana para la Investigación y el Desarrollo Educativo universitarios [Internet]. 2016 [consultado Marzo 2020]; 6(12):1-14. Disponible en: https://www.ride.org.mx/index.php/RIDE/article/view/213/952

30. Strunin L, Díaz LR, Díaz A, Heeren T, Winter M, Kuranz S, Hernandez CA, Fernández H, Solís C. Parental monitoring and family relations: associations with drinking patterns among male and female Mexican students. Addictive behaviors [Internet]. 2015 [consultado Marzo 2020]; 51(1): 143-151. Disponible en: https://doi.org/10.1016/j.addbeh.2015.07.025 31. Albarracín M, Muñoz L. Factores asociados al consumo de alcohol en estudiantes de los dos primeros años de carrera universitaria. Liberabit [Internet]. 2008 [consultado Marzo 2020]; 14(14): 49-61. Disponible en: http://www.scielo.org.pe/scielo.php?script=sci_arttext\&pid=S1729-48272008000100007\&lng=es\&tlng=es.

32. Castaño-Pérez GA, Calderón-Vallejo GA. Problemas asociados al consumo de alcohol en estudiantes universitarios. Rev. Latino-Am. Enfermagem [Internet]. 2014 [consultado Marzo 2020]; 22(55): 739-46. Disponible en: https://doi.org/10.1590/0104-1169.3579.2475

Cómo citar este artículo: Sifuentes-Castro JA, López-Cisneros MA, Guzmán-Facundo FR, Telumbre-Terrero JY, NohMoo PM. Rasgos de personalidad y consumo de alcohol en estudiantes universitarios. SANUS [Internet]. 2021 [citado el $\underline{\mathrm{dd}} \underline{\mathrm{mm}}$ aa];6:e194. Disponible en: DOI/Link 